Final Report

on

\title{
Coincidence Prompt Gamma-Ray Neutron Activation Analysis
}

(Grant No. FG07-99ID13775)

R.P. Gardner, C.W. Mayo, W.A. Metwally, W. Zhang, W. Guo, and A. Shehata

Center for Engineering Applications of Radioisotopes

Department of Nuclear Engineering

North Carolina State University

Raleigh, NC 27695-7909

Submitted to:

Nancy A. Elizondo

US Department of Energy

Idaho Operations Office

850 Energy Drive MS 1216

Idaho Falls, ID 83401-1563

e-mail: elizonna@id.doe.gov

November 10, 2002

Ref: neer06.tex 


\title{
OUTLINE
}

\author{
ABSTRACT \\ 1. INTRODUCTION \\ 2. MONTE CARLO SIMULATION OF COINCIDENCE COUNTING \\ 3. DETERMINING ELEMENTAL PROMPT GAMMA-RAY COINCIDENCE SCHEMES \\ 4. THE MONTE CARLO - LIBRARY LEAST-SQUARES (MCLLS) APPROACH \\ 5. CONCLUSIONS AND DISCUSSION \\ 6. FUTURE WORK \\ 7. REFERENCES
}

\begin{abstract}
The normal prompt gamma-ray neutron activation analysis for either bulk or small beam samples inherently has a small signal-to-noise $(\mathrm{S} / \mathrm{N})$ ratio due primarily to the neutron source being present while the sample signal is being obtained. Coincidence counting offers the possibility of greatly reducing or eliminating the noise generated by the neutron source. The present report presents our results to date on implementing the coincidence counting PGNAA approach. We conclude that coincidence PGNAA yields: (1) a larger signal-to-noise $(\mathrm{S} / \mathrm{N})$ ratio, (2) more information (and therefore better accuarcy) from essentially the same experiment when sophisticated coincidence electronics are used that can yield singles and coincidences simultaneously, and (3) a reduced (one or two orders of magnitude) signal from essentially the same experiment. In future work we will concentrate on: (1) modifying the existing CEARPGA Monte Carlo code to incorporate coincidence counting, (2) obtaining coincidence schemes for 18 or 20 of the common elements in coal and cement, and (3) optimizing the design of a PGNAA coincidence system for the bulk analysis of coal.
\end{abstract}




\section{INTRODUCTION}

Prompt gamma-ray neutron activation analysis (PGNAA) inherently has a low signalto-noise $(\mathrm{S} / \mathrm{N})$ ratio. This is primarily due to the fact that the exciting source of neutrons is present while the prompt gamma-ray signal is being collected. This leads to a number of noise (or background) sources that are not encountered in the normal neutron activation analysis (NAA) method. This includes: (1) the gamma rays associated with the neutron source, (2) the prompt gamma rays produced in the surrounding materials of the analyzer or sample holder, and (3) the prompt and other gamma rays produced in the detectors due to neutron activation or interaction. The use of coincidence counting in PGNAA greatly reduces or eliminates entirely all of these additional sources of background.

Therefore, the use of coincidence counting in PGNAA offers the possibility of greatly increasing the $\mathrm{S} / \mathrm{N}$ ratio by greatly reducing the noise. (It turns out that all elements except hydrogen emit prompt gamma rays in coincidence.) Unfortunately, the normal straightforward application of coincidence counting with the most common radiation detection systems also greatly reduces the signal due to the response being proportional to the product of detector solid angles. However, use of more sophisticated coincidence electronics allows one to obtain the normal (non-coincidence) spectral data as well as the coincidence spectral data, thereby guaranteeing that the accuracy obtainable be increased for otherwise identical radiation detection systems since more information is available from the same detection experiment. Also one may employ radiation detection systems that are optimized for coincidence counting that increase the coincidence signal that can be obtained. For example, the "trigger" detector used can be very large with much poorer resolution than normal. Multiple detector arrays also add to the coincidence detection probability (particularly when more than two prompt gamma rays are in coincidence) in excess of the total solid angle subtended as well as providing increasing detector locations to enhance the use of emission angular correlation.

Unfortunately, the fullest implementation of PGNAA coincidence counting requires additional knowledge that is not presently available - namely the prompt gamma-ray coincidence schemes themselves. This is true for all but a few elements like $\mathrm{H}, \mathrm{C}, \mathrm{Li}$, etc. that have simple enough schemes that they can be determined a priori. The vast majority of the elements emit many prompt gamma rays - many emit hundreds and the schemes are not

now known. This means that the full implementation of coincidence counting PGNAA will require that the coincidence schemes of all the elements of interest be determined. This will involve: (1) identifying the possible decay schemes, (2) determining the abundance of each scheme, and (3) determining the angular correlation between the prompt gamma rays within each scheme. While one could apply the library least-squares (LLS) analysis approach by a relatively straightforward experimental approach, this would not be the optimum approach that could be achieved if all the coincidence scheme information is available. The purely experimental approach would require that each experimental arrangement have all libraries evaluated at all sample compositions (the inverse problem is nonlinear) to be encountered 
- a very time consuming and tedious job. This would involve "spiking" the sample with each element of interest and then subtracting one large set of numbers (a spectrum) from another, which always represents a difficult statistical problem. Obviously it would be very difficult to use the purely experimental approach for optimizing the measurement system design. On the other hand, if the coincidence schemes of the elements of interest are known, one could easily use Monte Carlo simulation for measurement system design.

There is another compelling reason for determining all the pertinent elemental coincidence schemes. At the present time there is no way to account for the "summing" of prompt gamma rays in the same detector, because the coincidence schemes are unknown. These corrections can presently be made in NAA applications because most of the coincidence schemes for radioisotope decay gamma rays are well known. These summing corrections could be considerable for prompt gamma rays and should be accounted for. It is very likely that the present data on individual prompt gamma-ray abundances has been "clouded" by the fact that coincidence counting has not been employed in the experiments and some of the reported prompt gamma rays may either be entirely "sums" or at least partial sums so that reported abundances may be in considerable error.

In the present report we describe some preliminary results for applying coincidence counting to PGNAA. This includes: (1) the Monte Carlo simulation of PGNAA (the CEARPGA code) and coincidence PGNAA (the CEARCPG code), (2) the determination of elemental prompt gamma-ray coincidence schemes, (3) application of the Monte Carlo - library leastsquares (MCLLS) approach to coincidence PGNAA, (4) conclusions and discussion, and (5) future work.

\section{MONTE CARLO SIMULATION OF COINCIDENCE COUNTING}

We at the Center for Engineering Applications of Radioisotopes (CEAR) have previously developed a specific purpose Monte Carlo code called CEARPGA for simulating prompt gamma-ray neutron activation analysis (PGNAA). This code has been under continuous development since 1978. It began with the MS thesis of Clark (1981) and continued with the PhD theses of Yuan (1984), Jin (1886), Choi (1987), and Shyu (1991). Direct significant contributions were also made by Research Associates P. Guo and L. Liu. Indirect contributions to this code were made by PhD students and Research Associates that worked primarily on the CEARXRF (X-ray fluorescence code) Monte Carlo code. W. Zhang is presently working on his PhD thesis on improvements for the CEARPGA code.

The Monte Carlo - library least-squares (MCLLS) approach for handling the inverse problem of PGNAA essentially consists of using Monte Carlo simulation to generate the necessary libraries for the linear library least-squares analysis approach. This is discussed in more detail in a later section. The CEARPGA code has been used to implement the MCLLS algorithm for PGNAA coal sample analysis (Shyu, 1991 and Shyu, Gardner, and Verghese, 1993). The code incorporated a variety of variance reduction techniques to improve 
calculation efficiency such as forcing all prompt gamma rays to be emitted after a neutron interaction (stratified sampling), using the expected value splitting (EVS) technique to increase the score probability of each tracked gamma ray, using the correlated sampling method to deal with small variations of sample compositions, and using a detector response function to convert the incident gamma-ray spectra on the detector to pulse height spectra. While this code was efficient and accurate, it suffered from the big weight problem in which a few histories yield very large weights with very large variances. The big weight problem was found to be caused primarily by the expected value splitting technique used for tracking gamma rays (Shyu, 1991 and Zhang, 2002).

In order to minimize or eliminate this big weight problem while still maintaining high calculational efficiency, the authors have investigated two approaches: (1) the score importance map approach combined with the batch tracking technique and (2) the analog linear interpolation approach (ALI). Initial studies showed that both approaches are effective, but the ALI was judged to be better and has been implemented in the new modified CEARPGA code.

The ALI approach (Zhang, 2002) is a combination of the analog Monte Carlo simulation method and a linear interpolation technique and is mainly employed to track prompt gamma rays emitted from neutron capture interactions in the sample. In this approach a set of pseudo gamma rays that represent the actual prompt gamma rays are first tracked by analog Monte Carlo simulation to establish a set of energy-score tables. Later the linear interpolation approach is used to derive any incident prompt gamma-ray spectrum of interest, based on the energy-score tables. The procedure of implementing the ALI approach consists of the following steps:

(1) Choosing the number and energy of the pseudo gamma rays

Instead of tracking all prompt gamma rays emitted from the neutron capture interactions in the sample of interest (typical samples may have more than 2000 prompt gamma rays), only a set of pseudo gamma rays are selected and tracked as representatives of all the prompt gamma rays. The pseudo gamma rays should cover the energy range of the actual prompt gamma rays so that interpolation can be used later.

(2) Determining the number of gamma rays to sample

The number of gamma rays to sample of each energy is determined by trial and error to give optimum statistics.

(3) Tracking pseudo gamma rays

Each pseudo gamma ray is tracked starting from the site of the current neutron radiative capture interaction by the analog Monte Carlo approach, except that the photoelectric 
absorption is sampled implicitly.

(4) Tallying the scores of pseudo gamma rays

The magnitude of the score that a pseudo gamma ray has is the product of its weight accumulated along its track to the detector and the detector efficiency. The scores of incident gamma rays are recorded to a set of energy-score tables based on both pseudo gamma-ray energy and the incident energy. Therefore, the energy-score tables are of two dimensions, with one dimension representing the energy of incident gamma rays and the other representing the total scores of incident gamma rays in the previous histories. The score scheme for pair production annihilation photons takes into account the cases that both photons score simultaneously as well as each independently.

(5) Calculating average neutron capture macroscopic cross sections

For each element in the sample, the average neutron capture macroscopic cross section needs to be calculated during the process of simulation and is used later to adjust its interpolated incident gamma-ray spectrum. For the ith element in the sample region, the average cross section, $\mu_{(n, r)}^{i}$, is calculated according to the following formula:

$$
\mu_{(n, r)}^{i}=\frac{\sum_{j} w_{j}^{i} \mu_{j}^{i}}{\sum_{j} w_{i}^{j}}
$$

where $\mu_{j}^{i}$ is the neutron radiative capture macroscopic cross section of element $i$ for the $j t h$ neutron capture interaction in the sample, $w_{j}^{i}$ is the weight factor of the neutron in the neutron capture interaction.

(6) Interpolating the spectra for all gamma rays of interest

Incident spectra of gamma rays resulting from neutron capture interactions and radioisotope decays in the sample are obtained through linear interpolation at the end of the entire Monte Carlo simulation when a set of energy-score tables has been established. For the full energy channel of the gamma ray to be interpolated, the scores from the corresponding full energy channels of the pseudo gamma rays are used for interpolation. For other channels, the scores from the same channels are used.

The details of the modified CEARPGA code are given in an upcoming paper (Zhang and Gardner, 2002). Typical results of this code are shown in Fig. 1.

At present the CEARPGA code does not contain the capability to simulate the summing of coincident prompt gamma rays in the detector. This is being added to the code, but actual implementation must, of course, await the coincidence schemes that are needed. When these become available we will add the capability of sampling from the coincidence schemes rather than using the present necessary practice of sampling from the abundance of 
individual prompt gamma rays.

We believe that it will be very easy to modify the existing ALI-based CEARPGA code to include PGNAA coincidence counting. One will simply have to accumulate energy-score tables for two (or more) detectors rather than the present one. Then the coincident prompt gamma-ray spectra can be obtained by simply multiplying the probabilities of the two score tables to obtain the weight of the pertinent two energies in the score tables that are simply added together. We are investigating this approach at the present time.

\section{DETERMINING ELEMENTAL PROMPT GAMMA-RAY COINCIDENCE SCHEMES}

While a few of the elemental coincidence schemes are simple enough that they can be deduced directly from the known individual gamma-ray abundances, the majority are very complex with large numbers of prompt gamma rays. There is considerable information available for determining these schemes, including compilations and evaluations of experimental data on individual prompt gamma rays (Lone, Leavitt, and Harrison, 1981; Reedy and Frankel, 2002) and information based on theoretical considerations (Firestone, 1996). However, there is also considerable data that is not available, such as information on angular correlation. Also the existing experimental data on abundances may contain inaccuracies particularly since summing effects have probably not been taken into account.

The prompt gamma-ray coincidence schemes and their abundances for naturally abundant elements can be determined directly by taking experimental three-dimensional coincidence data on pure elemental samples. Or one can deduce the schemes by using existing data on individual gamma-ray energies, the total emitted energy (the $\mathrm{S}_{n}$ value) for the element of interest, and the individual gamma-ray abundances. ${ }^{1}$ The possible coincidence schemes can be deduced by first finding all the possible sets of gamma-ray energies that add up to the $\mathrm{S}_{n}$ value. This can be done either deterministically or by Monte Carlo simulation. Then one can determine the abundance of each identified scheme by an appropriate leastsquares or trial-and-error method that minimizes the sum of the differences squared between the nominal abundance of each individual prompt gamma ray and that calculated from the coincidence scheme sets. A useful mathematical representation of this problem is given by:

$$
A_{i}=\sum_{j=1}^{m} x_{i j} a_{j}+E_{i} \text { for } i=1, n
$$

where $A_{i}$ is the nominal (experimental) value of the abundance of the $i t h$ prompt gamma ray, $a_{j}$ is the abundance of the $j t h$ prompt gamma-ray coincidence scheme, $x_{i j}$ is a parameter (either 0 or 1 ) that determines if the ith gamma ray is present or not in the jth prompt gamma-ray coincidence scheme, and $E_{i}$ is the random error in the modeling of the

\footnotetext{
${ }^{1}$ Note that one must treat each stable isotope of each element separately as each isotope will have its own characteristic $S_{n}$ value and its own individual prompt gamma-ray coincidence sets.
} 
ith prompt gamma-ray abundance. Best policy is to form the reduced chi-square value $\left(\chi_{\nu}^{2}\right)$ for subsequent minimization.

$$
\chi_{\nu}^{2}=\sum_{i=1}^{n} E_{i}^{2} /\left[\sigma_{i}^{2}(n-m-1)\right]
$$

where $\sigma_{i}$ is the standard deviation of the $i t h$ prompt gamma-ray abundance.

When $n$ exceeds $m$, one can use the linear least-squares approach which is obtained by partially differentiating $\chi_{\nu}^{2}$ with respect to each prompt gamma-ray coincidence scheme.

$$
\partial \chi_{\nu}^{2} / \partial a_{k}=2 \sum_{i=1}^{n}\left(A_{i}-\sum_{j=1}^{m} x_{i j} a_{j}\right) x_{i k}=0 \text { for } k=1, m
$$

This results in $m$ equations in $m$ unknowns (the $a_{j}$ ) that can be solved by any appropriate linear method of simultaneous solution.

In those cases when $m$ exceeds $n$ we are investigating the use of linear programming methods.

We have determined preliminary prompt coincidence schemes and their abundances for several elements including the simple ones like hydrogen, carbon, oxygen, etc. and several elements of intermediate difficulty including sulfur and nitrogen. We are also beginning to investigate more complex elements of interest in coal and cement bulk analysis including chlorine, aluminum, iron, and other common metals.

Two elements of particular interest are sodium and iodine since they are the two major elements in the most commonly used radiation detector (NaI). Their coincidence schemes are needed for predicting the prompt gamma-ray response that occurs when neutrons enter this detector and interact with it to produce the sums and partial sums of the gamma-ray energies in the various prompt (and radioisotope) coincidence schemes. Iodine is the most important since it has a thermal neutron activation cross section of 6.2 barns while that of sodium is only 0.40 barns. Fortunately, iodine has only one stable isotope. Unfortunately, it emits more than 400 prompt gamma rays.

\section{THE MONTE CARLO - LIBRARY LEAST-SQUARES (MCLLS) APPROACH}

Development of the MCLLS approach was begun by C.M. Shyu in his $1991 \mathrm{PhD}$ thesis for PGNAA and by T. He for Energy-Dispersive X-Ray Fluorescence (EDXRF) Analysis in his $1992 \mathrm{PhD}$ thesis. Both analysis methods are nonlinear in that the detected response for a specific element depends on all elements in the sample. By assuming a sample composition, elemental libraries can be calculated by Monte Carlo simulation for use in a linear analysis. If the calculated values are too far from the assumed, iterations on sample composition are 
made until linearity is obtained.

Several complications arose in applying the PGNAA MCLLS approach including the nonlinearity of the large $\mathrm{NaI}$ detectors that are used in commercial practice and the background that is introduced by the neutron activation of these detectors. Gardner and Mayo (1999) treated NaI nonlinearity while E.S. El Sayyed treated the NaI neutron activation problem in his $2001 \mathrm{MS}$ and in an article ( Gardner, et al.,2000) with others. The NaI nonlinearity problem has subsequently been treated in a much better way (Gardner ans Sood, 2002) by including it in a detector response function treatment.

As discussed previously the PGNAA approach has a notoriously small signal-to-noise ratio due to its inherently high background from hydrogen prompt gamma rays, the prompt and radioisotope gamma rays from construction materials, the gamma rays from the Cf-252 radioisotope neutron source, and the neutron activation of the $\mathrm{NaI}$ detectors that are often used. All of these sources of background can be eliminated or greatly minimized by the use of the coincidence counting of the signal (sample) prompt gamma rays.

Since commercial PGNAA devices often use two large NaI detector systems already, adding coincidence counting only involves adding the coincidence electronics. In the present case we choose to add a sophisticated coincidence system that obtains the singles and coincidence counts simultaneously. In this way one obtains the coincidence counts in addition to the normal singles counts, so with this added information from the same counting time and device hardware, one must obtain greater accuracy.

We also intend to use the MCLLS approach with the coincidence approach. Preliminary results have been obtained with the three radioisotopes Cs-134, Co-60, and Na-24 that all emit coincident gamma rays. This work is reported in a paper to be published soon (Metwally, Gardner, and Mayo, 2002). Basically it was found in that work that the coincidence MCLLS is more resistant to pulse pile-up problems than the normal non-coincident approach in that it gives better accuracy.

Application of the library least-squares (LLS) approach to coincident prompt (or other) gamma rays is very straightforward and can be done in the same fashion as that for noncoincident gamma rays. A possible improvement in the coincident application is the use of three-dimensional data such that the reduced chi-square is formed from a matrix of data rather than the normal vector approach. The normal reduced chi-square is given by:

$$
\chi_{\nu}^{2}=\sum_{i=1}^{n}\left(E_{i}-M_{i}\right)^{2} /\left[\sigma_{i}^{2}(n-m-1)\right]
$$

where $E_{i}$ and $M_{i}$ are the experimental and model $i t h$ data points, $\sigma_{i}$ is the standard deviation of the $i t h$ data point, and $n$ and $m$ are the number of data points and number of model parameters, respectively. For the three-dimensional coincidence case the reduced chi-square is given by: 


$$
\chi_{\nu}^{2}=\sum_{i=1}^{n} \sum_{j=1}^{n}\left(E_{i j}-M_{i j}\right)^{2} /\left[\sigma_{i j}^{2}\left(n^{2}-m-1\right)\right]
$$

where the $E_{i j}$ and $M_{i j}$ are functions of the gamma-ray energies in detector $1(i)$ and $2(j)$, respectively. Potentially there is more information in using this approach in that there may be more information content in this matrix representation. We are looking into this possibility.

\section{CONCLUSIONS AND DISCUSSION}

Coincidence counting in PGNAA yields: (1) a larger signal-to-noise (S/N) ratio, (2) more information (and therefore better accuarcy) from essentially the same experiment when sophisticated coincidence electronics are used that can yield singles and coincidences simultaneously, and (3) a reduced (one or two orders of magnitude) signal from essentially the same experiment. To effect the maximum benefit from PGNAA coincidence counting it will be necessary to design systems that optimize the coincidence signal. This may include the use of very large "trigger" detectors, or multiple detectors, or both. Modification of the CEARPGA Monte Carlo code to include coincidence counting (the CEARCPG code) will be a major contribution that will allow the practical optimization of coincidence systems.

\section{FUTURE WORK}

In the future our work will concentrate on: (1) modifying the existing CEARPGA Monte Carlo code to incorporate coincidence counting, (2) obtaining coincidence schemes for 18 or 20 of the common elements in coal and cement, and (3) optimizing the design of a PGNAA coincidence system for the bulk analysis of coal.

\section{REFERENCES}

- Choi, H.K., 1987, "Monte Carlo Simulation of the Temporal and Spectral Responses of the Pulsed Neutron Logging Principle", Ph.D. Thesis, Department of Nuclear Engineering, North Carolina State University, Raleigh, NC.

- Clark, T.C., 1981, “A Monte Carlo Model for Prompt Gamma-Ray Systems of Interest to the Mining Industries", M.S. Thesis, Department of Nuclear Engineering, North Carolina State University, Raleigh, NC.

- Firestone, R.B., 1996, TABLE OF ISOTOPES, 8th Edition, John Wiley \& Sons, Inc., New York.

- Gardner, R.P. and C.W. Mayo, 1999, "NaI Detector Nonlinearity for PGNAA Applications", Applied Radiation and Isotopes, Vol. 51, pp. 189-195. 
- Gardner, R.P., C.W. Mayo, E.S. El Sayyed, W. Zhang, 2000, "Software Progress in the PGNAA of Bulk Materials", Transactions of the American Nuclear Society, Vol. 82, pp. 99-100.

- Gardner, R.P. and A. Sood, 2002, “A Monte Carlo Simulation Approach for Generating NaI Detector Response Functions (DRF's) that Accounts for Nonlinearity and Variable Flat Continua", submitted to Proceedings of IRRMA-V to be published in Nuclear Instruments and Methods B.

- Jin, Y., 1986, "Monte Carlo Simulation of the Complete Spectral Response of Prompt Gamma Ray Analysis Systems", Ph.D. Thesis, Department of Nuclear Engineering, North Carolina State University, Raleigh, NC.

- Lone, M.A., R.A. Leavitt, and D.A. Harrison, 1981, "Prompt Gamma Rays from Thermal Neutron Capture", Atomic Data and Nuclear Data Tables, 26, 511-559.

- Metwally, W.A., R.P. Gardner, and C.W. Mayo, 2002, "Elemental PGNAA Analysis using Gamma-Gamma Coincidence Counting with the Library Least-Squares (LLS) Approach", submitted to Proceedings of IRRMA-V to be published in Nuclear Instruments and Methods B.

- Reedy, R.C. and S.C. Frankel, 2002, "Prompt Gamma Rays from Radiative Capture of Thermal Neutrons by Elements from Hydrogen through Zinc", Atomic Data and Nuclear Data Tables, 80, 1-34.

- Shyu, C.M., 1991, "Development of the Monte Carlo - Library Least-Squares Method of Analysis for Neutron Capture Prompt Gamma-Ray Analyzers", Ph.D. Thesis, Department of Nuclear Engineering, North Carolina State University, Raleigh, NC.

- Shyu, C.M., R.P. Gardner, and K. Verghese, 1993, "Development of the Monte Carlo Library Least-Squares Method of Analysis for Neutron Capture Prompt Gamma-Ray Analyzers", Nuclear Geophysics, Vol. 7, No. 2, pp. 241-268.

- Yuan, R.Y., 1984, "A Monte Carlo Model for Neutron Capture Prompt Gamma-Ray Analysis of Coal in Transmission Geometry", Ph.D. Thesis, Department of Nuclear Engineering, North Carolina State University, Raleigh, NC.

- Zhang, W., 2002, "The Analog Linear Interpolation Approach for Monte Carlo Simulation of Prompt Gamma-Ray Neutron Activation Analysis", Ph.D. Thesis, Department of Nuclear Engineering, North Carolina State University, Raleigh, NC.

- Zhang, W. and R.P. Gardner, 2002, "The Analog Linear Interpolation Approach for Monte Carlo Simulation of PGNAA: The CEARPGA Code", submitted to Proceedings of IRRMA-V to be published in Nuclear Instruments and Methods B. 
Fig. 1. Comparison of CEARPGA Monte Carlo simulation and experiment

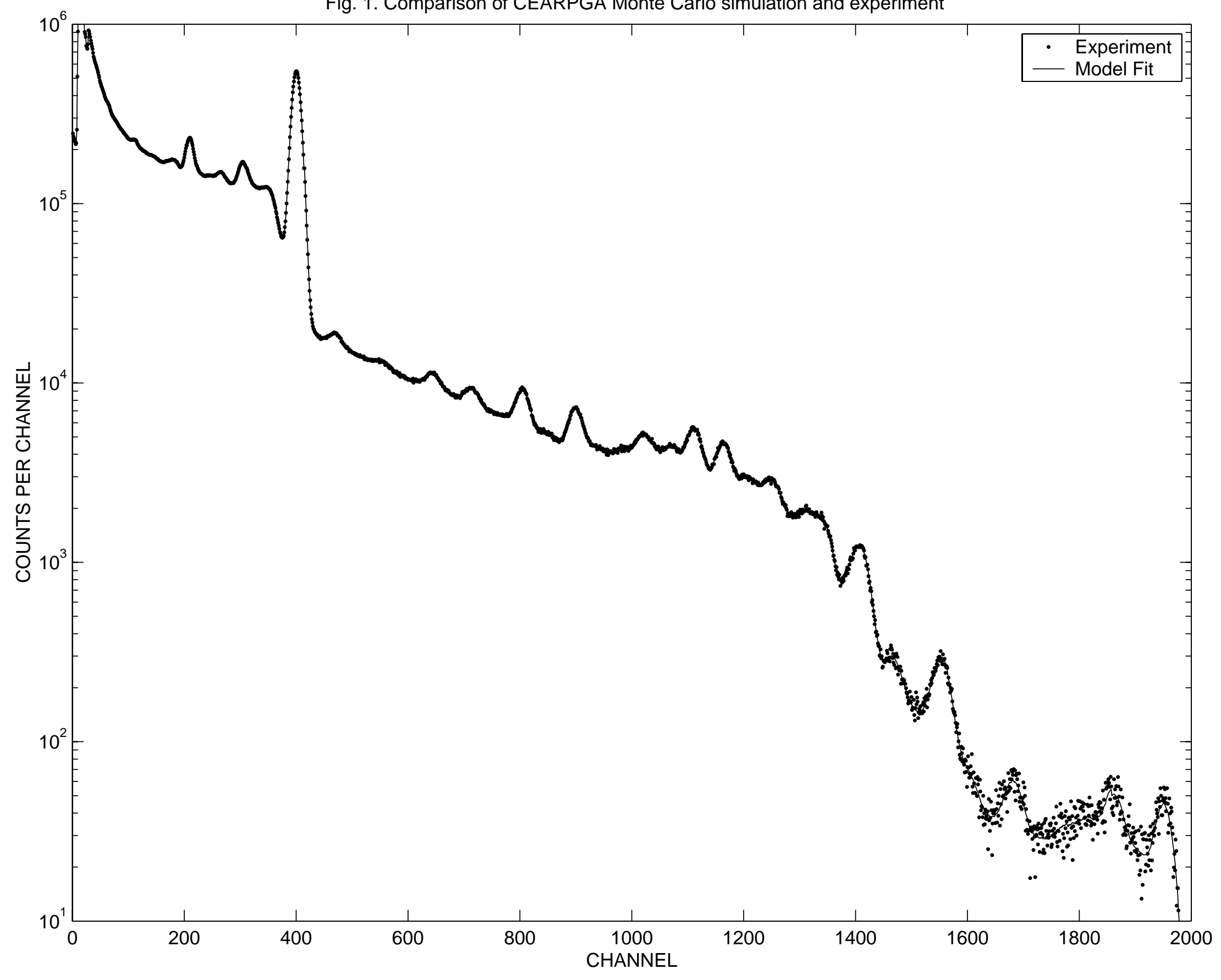

